\title{
ZERO-NONZERO PATTERNS FOR NILPOTENT MATRICES OVER FINITE FIELDS*
}

\author{
KEVIN N. VANDER MEULEN ${ }^{\dagger}$ AND ADAM VAN TUYL $\ddagger$
}

\begin{abstract}
Fix a field $\mathbb{F}$. A zero-nonzero pattern $\mathcal{A}$ is said to be potentially nilpotent over $\mathbb{F}$ if there exists a matrix with entries in $\mathbb{F}$ with zero-nonzero pattern $\mathcal{A}$ that allows nilpotence. In this paper an investigation is initiated into which zero-nonzero patterns are potentially nilpotent over $\mathbb{F}$ with a special emphasis on the case that $\mathbb{F}=\mathbb{Z}_{p}$ is a finite field. A necessary condition on $\mathbb{F}$ is observed for a pattern to be potentially nilpotent when the associated digraph has $m$ loops but no small $k$-cycles, $2 \leq k \leq m-1$. As part of this investigation, methods are developed, using the tools of algebraic geometry and commutative algebra, to eliminate zero-nonzero patterns $\mathcal{A}$ as being potentially nilpotent over any field $\mathbb{F}$. These techniques are then used to classify all irreducible zero-nonzero patterns of order two and three that are potentially nilpotent over $\mathbb{Z}_{p}$ for each prime $p$.
\end{abstract}

Key words. Zero-nonzero patterns, Nilpotent, Ideal saturation, Gröbner basis, Finite fields.

AMS subject classifications. 15A18, 13P10, 05C50, $11 \mathrm{~T} 06$.

1. Introduction. A zero-nonzero (znz) pattern $\mathcal{A}$ is a square matrix whose entries come from the set $\{*, 0\}$ where $*$ denotes a nonzero entry. Fix a field $\mathbb{F}$. We then set

$$
Q(\mathcal{A}, \mathbb{F})=\left\{A \in M_{n}(\mathbb{F}) \mid(A)_{i, j} \neq 0 \Leftrightarrow(\mathcal{A})_{i, j}=* \text { for all } i, j\right\} .
$$

The set $Q(\mathcal{A}, \mathbb{F})$, sometimes denoted $Q(\mathcal{A})$ when $\mathbb{F}$ is known, is usually called the qualitative class of $\mathcal{A}$. An element $A \in Q(\mathcal{A}, \mathbb{F})$ is called a matrix realization of $\mathcal{A}$. A znz-pattern $\mathcal{A}$ is said to be potentially nilpotent over $\mathbb{F}$ if there exists a matrix $A \in Q(\mathcal{A}, \mathbb{F})$ such that $A^{k}=0$ for some positive integer $k$. In this paper we study the question of what patterns $\mathcal{A}$ are potentially nilpotent over a field $\mathbb{F}$. Although we will present some results for arbitrary fields, we are particularly interested in the case that $\mathbb{F}$ is a finite field.

One motivation to study this question is to provide a step in understanding spectrally arbitrary patterns in the context of fields other than $\mathbb{R}$. An $n \times n$ znzpattern $\mathcal{A}$ is a spectrally arbitrary pattern (SAP) if given any monic polynomial $p(x)$ of

* Received by the editors December 18, 2008. Accepted for publication October 8, 2009. Handling Editor: Michael J. Tsatsomeros.

${ }^{\dagger}$ Department of Mathematics, Redeemer University College, Ancaster, ON L9K 1J4 Canada (kvanderm@redeemer.ca). Research supported in part by an NSERC Discovery Grant.

${ }_{\ddagger}^{\ddagger}$ Department of Mathematical Sciences, Lakehead University, Thunder Bay, ON P7B 5E1 Canada (avantuyl@lakeheadu.ca). Research supported in part by an NSERC Discovery Grant. 
degree $n$ with coefficients in $\mathbb{F}$, there exists a matrix $A \in Q(\mathcal{A}, \mathbb{F})$ whose characteristic polynomial is $p(x)$. Note that every SAP is potentially nilpotent. There is a growing body of literature (see, for example, $[2,5,7,18,19]$ and their references) interested in identifying SAPs when $\mathbb{F}=\mathbb{R}$ (with much focus on sign patterns: patterns whose entries come from the set $\{+,-, 0\})$. Recently, work has begun on the problem of identifying SAPs over finite fields [1] and over $\mathbb{C}$ [21].

We now cursorily survey the problem of identifying potentially nilpotent patterns over $\mathbb{F}=\mathbb{R}$. Determining when a sign pattern is potentially nilpotent was listed as an open problem in [9]. Potentially nilpotent star sign patterns were introduced in [20] and fully characterized in [17]. Potentially nilpotent sign patterns of order up to 3 were characterized in [10]. Included in [10] is an investigation of sign patterns that allow nilpotence of index 2 , where the index of matrix $A$ is the smallest integer $k$ such that $A^{k}=0$; this was later extended to index 3 in [11] (see also [3]). In [18], it was shown that all potentially nilpotent full sign patterns (i.e. patterns with no zero entries) are also SAPs. Consequently, recent work [15] presents constructions of potentially nilpotent full sign patterns. Much work in determining when a pattern is potentially nilpotent occurs in the literature on SAPs. Identifying potentially nilpotent patterns over $\mathbb{R}$ is in part an important subproblem in the study of SAPs due to a technique developed in [8], usually referred to as the Nilpotent-Jacobi Method. Roughly speaking, if $A$ is a nilpotent realization over $\mathbb{R}$ of a pattern $\mathcal{A}$, then one can determine if $\mathcal{A}$ is spectrally arbitrary by evaluating the entries of $A$ in a Jacobian matrix constructed from $\mathcal{A}$. Note that this technique requires the Implicit Function Theorem, which holds over $\mathbb{R}$, so one should not expect a generalization of this approach to finite fields.

We begin in Section 2 by reviewing some basic results concerning nilpotent matrices over a field $\mathbb{F}$. Many of the results that are known to hold in $\mathbb{R}$ continue to hold over an arbitrary field.

In Section 3 we introduce some techniques to eliminate certain patterns as being potentially nilpotent over a field. We use some tools from commutative algebra and algebraic geometry to carry out this program. Starting with a znz-pattern $\mathcal{A}$ with nonzero entries at $\left(i_{1}, j_{1}\right), \ldots,\left(i_{t}, j_{t}\right)$, we define an ideal $I_{\mathcal{A}}$ in a polynomial ring $R_{\mathcal{A}}=\mathbb{F}\left[z_{i_{1}, j_{1}}, \ldots, z_{i_{t}, j_{t}}\right]$ over the field $\mathbb{F}$. In Theorem 3.2 we show that $\mathcal{A}$ is potentially nilpotent over a field $\mathbb{F}$ if and only if a certain subset of the affine variety defined by $I_{\mathcal{A}}$ is nonempty. With this characterization, we can use the technique of ideal saturation (see Definition 3.4) to determine if a given pattern is not potentially nilpotent:

Theorem 3.5. Let $\mathbb{F}$ be any field and $\mathcal{A}$ a znz-pattern. Let $J=\left(z_{i_{1}, j_{1}} \cdots z_{i_{t}, j_{t}}\right)$ be the ideal generated by the product of the variables of $R_{\mathcal{A}}$. If $1 \in I_{\mathcal{A}}: J^{\infty}$, then $\mathcal{A}$ is not potentially nilpotent over any extension of $\mathbb{F}$. 
Since many computer algebra programs can compute the saturation of ideals, Theorem 3.5 promises to be a useful tool for future experimentation. In the last part of Section 3 we review the basics of Gröbner bases, and show how Gröbner bases can also be used to eliminate znz-patterns as being potentially nilpotent (see Example 3.13).

As an aside, we hope that our results, along with the work of Shader [19] and Kaphle [13], will highlight the usefulness of techniques from commutative algebra and algebraic geometry in the study of SAPs. Shader uses a result about the number of algebraically independent elements over the polynomial ring $\mathbb{R}\left[x_{1}, \ldots, x_{n}\right]$ to prove a lower bound on the number of nonzero entries in a SAP. Kaphle's MSc thesis uses Gröbner bases to eliminate sign patterns as being potentially nilpotent. Note that one difference between our work and the work of Kaphle is that we use the equations constructed from the characteristic polynomials when forming the Gröbner basis, while Kaphle uses equations constructed from the traces of the matrices $\mathcal{A}^{k}$ for $k=1, \ldots, n$.

In Section 4 we introduce a necessary condition for a znz-pattern $\mathcal{A}$ to be nilpotent over a field $\mathbb{F}$. Precisely, we look at znz-patterns $\mathcal{A}$ where $\mathcal{A}$ is irreducible and the digraph $D(\mathcal{A})$ has no 2-cycles (see Section 2). When $\mathbb{F}=\mathbb{R}$, if $\mathcal{A}$ has at least two nonzero entries on the diagonal and $\mathcal{A}$ is potentially nilpotent, then it is known (see $[7])$ that $D(\mathcal{A})$ must to have a 2-cycle. We explore the fact that this is no longer true over an arbitrary field: what is important is that the polynomial $x^{3}-1$ factors completely over $\mathbb{F}$. In fact, we prove a more general result:

Theorem 4.4. Let $\mathcal{A}$ be a znz-pattern with $m \geq 2$ nonzero entries on the diagonal, and suppose that $D(\mathcal{A})$ has no $k$-cycles with $2 \leq k \leq m-1$. If $\mathcal{A}$ is potentially nilpotent over $\mathbb{F}$, then the polynomial $x^{m}-1$ factors into $m$ linear forms over $\mathbb{F}$.

Our paper culminates with Section 5 which uses the above techniques to classify all potentially nilpotent patterns of order at most three when $\mathbb{F}=\mathbb{Z}_{p}$ is the finite field with $p$ elements, where $p$ is a prime (see Theorems 5.1 and 5.3). One interesting by-product of this classification is the discovery that $\mathcal{A}$ may be potentially nilpotent in a field $\mathbb{F}$, but a superpattern of $\mathcal{A}$, that is, a znz-pattern $\mathcal{A}^{\prime}$ such that $\left(\mathcal{A}^{\prime}\right)_{i, j} \neq 0$ whenever $(\mathcal{A})_{i, j} \neq 0$, might not be potentially nilpotent over the same field $\mathbb{F}$.

2. Basic Properties. In this section, we review some of the needed properties of znz-pattern matrices and summarize some of the basic properties of potentially nilpotent matrices over $\mathbb{F}$. Some of these results were known when $\mathbb{F}=\mathbb{R}$; we consider the more general case. We continue to use the notation from the introduction.

When referring to elements of the field $\mathbb{F}$, we shall use $1_{\mathbb{F}}$ to denote the multiplicative identity of $\mathbb{F}$, but abuse notation slightly and write 0 for the additive identity $0_{\mathbb{F}}$. 
For any positive integer $n \in \mathbb{Z}$, we write $n_{\mathbb{F}}$ to denote $\left(1_{\mathbb{F}}+\cdots+1_{\mathbb{F}}\right)$ ( $n$ times). Then $-n_{\mathbb{F}}$ will denote the additive inverse of $n_{\mathbb{F}}$ in $\mathbb{F}$, and $n_{\mathbb{F}}^{-1}$ denotes the multiplicative inverse (provided $n_{\mathbb{F}} \neq 0$ ).

Given an $n \times n$ znz-pattern $\mathcal{A}$, we can construct a digraph $D(\mathcal{A})=(V, E)$ on the vertex set $V=[n]:=\{1, \ldots, n\}$, whose edge set consists of the $\operatorname{arcs}(i, j)$ whenever $(\mathcal{A})_{i, j} \neq 0$. We call the edge $(i, i)$ a loop; loops correspond to the nonzero entries on the diagonal of $\mathcal{A}$. A simple cycle $\gamma$ of length $k$, also called a $k$-cycle, is a sequence of $k$ distinct vertices $\left\{i_{1}, \ldots, i_{k}\right\}$ such that $\left(i_{1}, i_{2}\right),\left(i_{2}, i_{3}\right), \ldots,\left(i_{k-1}, i_{k}\right),\left(i_{k}, i_{1}\right) \in E$. We sometimes denote a $k$-cycle $\gamma$ by $\left(i_{1}, i_{2}, \ldots, i_{k}\right)$, and denote its length by $|\gamma|=k$. Furthermore, we say two cycles $\gamma_{1}$ and $\gamma_{2}$ are disjoint if they have no vertices in common.

Suppose that $A \in Q(\mathcal{A}, \mathbb{F})$ is a realization of $\mathcal{A}$. The characteristic polynomial of $A$ can be described in terms of the cycles of $D(\mathcal{A})$. Precisely, suppose that $\gamma=$ $\left(i_{1}, \ldots, i_{k}\right)$ is a $k$-cycle. We let $\prod(\gamma)=a_{i_{1}, i_{2}} a_{i_{2}, i_{3}} \cdots a_{i_{k}, i_{1}}$ where $a_{i, j}=(A)_{i, j}$. Then the characteristic polynomial of $A$ has the form

$$
p_{A}(x)=x^{n}+r_{1} x^{n-1}+r_{2} x^{n-2}+\cdots+r_{n-1} x+r_{n}
$$

with

$$
r_{i}=(-1)^{i} \sum\left[\left((-1)^{\left|\gamma_{1}\right|-1} \prod\left(\gamma_{1}\right)\right) \cdots\left((-1)^{\left|\gamma_{p}\right|-1} \prod\left(\gamma_{p}\right)\right)\right] .
$$

where the sum is over all pairwise disjoint cycles $\gamma_{1}, \ldots, \gamma_{p}$ such that $\left|\gamma_{1}\right|+\cdots+\left|\gamma_{p}\right|=i$.

A znz-pattern $\mathcal{A}$ of order $n \geq 2$ is reducible if there exists some integer $1 \leq r \leq$ $n-1$ and a permutation matrix $P$ such that

$$
P \mathcal{A} P^{T}=\left[\begin{array}{cc}
\mathcal{A}_{1} & \mathcal{A}_{2} \\
0_{r, n-r} & \mathcal{A}_{3}
\end{array}\right] .
$$

Otherwise, a znz-pattern $\mathcal{A}$ is called irreducible. Equivalently, a znz-pattern $\mathcal{A}$ is irreducible if and only if the associated digraph $D(\mathcal{A})$ is strongly connected, that is, there is a directed path between any pair of distinct vertices. The Frobenius normal form of $\mathcal{A}$ is a permutation similar block upper triangular matrix whose diagonal blocks are irreducible. The diagonal blocks are called the irreducible components of $\mathcal{A}$.

The final lemma of this section summarizes some of the results we will need in the later sections.

Lemma 2.1. Fix a field $\mathbb{F}$ and a znz-pattern $\mathcal{A}$.

(a) Suppose that $\mathcal{A}$ is reducible with irreducible components $\mathcal{A}_{1}, \ldots, \mathcal{A}_{t}$. Then $\mathcal{A}$ is potentially nilpotent over $\mathbb{F}$ if and only if each znz-pattern $\mathcal{A}_{i}$ is potentially nilpotent. 
(b) If $\mathcal{A}$ is potentially nilpotent over $\mathbb{F}$, then so is $\mathcal{A}^{T}$, the transpose of $\mathcal{A}$.

(c) If $A$ is a nilpotent realization of $\mathcal{A}$, then the characteristic polynomial of $A$ is $p_{A}(x)=x^{n}$.

3. Eliminating potentially nilpotent candidates via ideal saturation. Let $\mathbb{F}$ be any field. Using some tools and techniques from commutative algebra and algebraic geometry, we will show that a znz-pattern $\mathcal{A}$ is potentially nilpotent over $\mathbb{F}$ if and only if a certain geometric set is nonempty. As an application, we develop an algebraic method to eliminate certain znz-patterns $\mathcal{A}$ as being potentially nilpotent over $\mathbb{F}$. We also explain how to use Gröbner bases to show that some patterns are not potentially nilpotent. While we will endeavor to keep this material as self-contained as possible, further background material can be found in the book of Cox, Little, and O'Shea $[6]$.

We begin with some notation. Fix a znz-pattern $\mathcal{A}$, and let $S_{\mathcal{A}}=\left\{(i, j) \mid(\mathcal{A})_{i, j} \neq\right.$ $0\}$ be the locations of the nonzero elements in $\mathcal{A}$. We then define the polynomial ring

$$
R_{\mathcal{A}}:=\mathbb{F}\left[z_{i, j} \mid(i, j) \in S_{\mathcal{A}}\right]=\mathbb{F}\left[z_{i_{1}, j_{1}}, \ldots, z_{i_{t}, j_{t}}\right]
$$

in $t=\left|S_{\mathcal{A}}\right|$ variables over the field $\mathbb{F}$. Associate to $\mathcal{A}$ the matrix $M_{\mathcal{A}}$ where

$$
\left(M_{\mathcal{A}}\right)_{i, j}:= \begin{cases}z_{i, j} & \text { if }(\mathcal{A})_{i, j} \neq 0 \\ 0 & \text { if }(\mathcal{A})_{i, j}=0\end{cases}
$$

Note that $M_{\mathcal{A}}$ is not a realization of $\mathcal{A}$ since the entries of $M_{\mathcal{A}}$ are variables. The characteristic polynomial of $M_{\mathcal{A}}$ then has the form

$$
p_{M_{\mathcal{A}}}(x)=x^{n}-F_{1} x^{n-1}+F_{2} x^{n-2}+\cdots+(-1)^{n-1} F_{n-1} x+(-1)^{n} F_{n}
$$

where each coefficient $F_{i}=F_{i}\left(z_{i_{1}, j_{1}}, \ldots, z_{i_{t}, j_{t}}\right)$ is a polynomial in $R_{\mathcal{A}}$. We then use the $n$ coefficients of the characteristic polynomial to define an ideal of $R_{\mathcal{A}}$. Precisely, let

$$
I_{\mathcal{A}}:=\left(F_{1}, \ldots, F_{n}\right) \subseteq R_{\mathcal{A}} .
$$

In fact, $I_{\mathcal{A}}$ is a homogeneous ideal since for each $F_{i} \neq 0$, the polynomial $F_{i}$ is a homogeneous polynomial of degree $i$; recall that a polynomial $G$ is homogeneous if each term of $G$ has the same degree. To see this fact, note that each term of $F_{i}$ corresponds to a composite cycle of length $i$ in the directed graph $D(\mathcal{A})$ (see the formula in Section 2), from which it follows that $F_{i}$ is homogeneous. Hence, every znz-pattern $\mathcal{A}$ induces a homogeneous ideal $I_{\mathcal{A}}$.

EXAMPLE 3.1. We illustrate the above notation with the following znz-pattern

$$
\mathcal{A}=\left[\begin{array}{lll}
* & * & 0 \\
* & 0 & * \\
0 & * & *
\end{array}\right]
$$


The associated matrix is

$$
M_{\mathcal{A}}=\left[\begin{array}{ccc}
z_{1,1} & z_{1,2} & 0 \\
z_{2,1} & 0 & z_{2,3} \\
0 & z_{3,2} & z_{3,3}
\end{array}\right]
$$

where the $z_{i, j}$ 's are indeterminates in the polynomial ring

$$
R_{\mathcal{A}}=\mathbb{F}\left[z_{1,1}, z_{1,2}, z_{2,1}, z_{2,3}, z_{3,2}, z_{3,3}\right] .
$$

The ideal $I_{\mathcal{A}}$ is then generated by three homogeneous polynomials:

$$
I_{\mathcal{A}}=\left(z_{1,1}+z_{3,3}, \quad z_{1,2} z_{2,1}+z_{2,3} z_{3,2}+z_{1,1} z_{3,3}, \quad z_{1,1} z_{2,3} z_{3,2}+z_{1,2} z_{2,1} z_{3,3}\right) .
$$

For each $\underline{a}=\left(a_{i_{1}, j_{1}}, \ldots, a_{i_{t}, j_{t}}\right) \in \mathbb{F}^{t}$, let $M_{\mathcal{A}}(\underline{a})$ denote the matrix obtained by replacing each $z_{i_{k}, j_{k}}$ with $a_{i_{k}, j_{k}}$. The characteristic polynomial of $M_{\mathcal{A}}(\underline{a})$ will have the form:

$$
p_{M_{\mathcal{A}}(\underline{a})}(x)=x^{n}-F_{1}(\underline{a}) x^{n-1}+F_{2}(\underline{a}) x^{n-2}+\cdots+(-1)^{n-1} F_{n-1}(\underline{a}) x+(-1)^{n} F_{n}(\underline{a}) .
$$

If $\mathcal{A}$ is potentially nilpotent over $\mathbb{F}$, then there exists an $\underline{a} \in \mathbb{F}^{t}$ with all $a_{i, j} \neq 0$ such that $M_{\mathcal{A}}(\underline{a})$ is a nilpotent matrix. In particular, the characteristic polynomial of $M_{\mathcal{A}}(\underline{a})$ must be $x^{n}$ by Lemma 2.1 (c), which, in turn, implies that $F_{i}(\underline{a})=0$ for $i=1, \ldots, n$. Thus, one can determine if a znz-pattern $\mathcal{A}$ is potentially nilpotent over $\mathbb{F}$ if one understands the affine variety described by $I_{\mathcal{A}}$; the affine variety ${ }^{1}$ defined by $I_{\mathcal{A}}$ is the set

$$
\begin{aligned}
V\left(I_{\mathcal{A}}\right) & =\left\{\underline{a} \in \mathbb{F}^{t} \mid G(\underline{a})=0 \text { for all } G \in I_{\mathcal{A}}\right\} \\
& =\left\{\underline{a} \in \mathbb{F}^{t} \mid F_{1}(\underline{a})=\cdots=F_{n}(\underline{a})=0\right\} .
\end{aligned}
$$

The set $V\left(I_{\mathcal{A}}\right)$ contains all the elements $\underline{a} \in \mathbb{F}^{t}$ such that the matrix $M_{\mathcal{A}}(\underline{a})$ is nilpotent. Thus, if $\mathcal{A}$ is potentially nilpotent over $\mathbb{F}$ and $M_{\mathcal{A}}(\underline{a})$ is a realization of $\mathcal{A}$ that is nilpotent, then $\underline{a} \in V\left(I_{\mathcal{A}}\right)$. However, the converse is not necessarily true. Indeed, if $\underline{b} \in V\left(I_{\mathcal{A}}\right)$, then while $M_{\mathcal{A}}(\underline{b})$ still has a characteristic polynomial of $x^{n}$, the matrix $M_{\mathcal{A}}(\underline{b})$ may not be a realization of $\mathcal{A}$. As a simple example, note that $\underline{0}=(0, \ldots, 0) \in V\left(I_{\mathcal{A}}\right)$, (since each $F_{i}$ is homogeneous, and thus $F_{i}(\underline{0})=0$ for all $i$ ), but $M_{\mathcal{A}}(\underline{0})$ is the zero-matrix, which is not a realization of $\mathcal{A}$.

For each indeterminate $z_{i, j} \in R_{\mathcal{A}}$, let $V\left(z_{i, j}\right)$ denote the associated affine variety, that is, $V\left(z_{i, j}\right)=\left\{\underline{a} \in \mathbb{F}^{t} \mid a_{i, j}=0\right\}$. With this notation, we can determine if a znz-pattern $\mathcal{A}$ is potentially nilpotent over $\mathbb{F}$ :

\footnotetext{
${ }^{1}$ What we call an affine variety is sometimes called an algebraic set. We decided to follow [6].
} 
Theorem 3.2. Fix a field $\mathbb{F}$ and a znz-pattern $\mathcal{A}$. Then $\mathcal{A}$ is potentially nilpotent over $\mathbb{F}$ if and only if

$$
V\left(I_{\mathcal{A}}\right) \backslash \bigcup_{k=1}^{t} V\left(z_{i_{k}, j_{k}}\right) \neq \emptyset .
$$

Proof. If $\mathcal{A}$ is potentially nilpotent over $\mathbb{F}$, then there exists an $\underline{a} \in \mathbb{F}^{t}$ such that $M_{\mathcal{A}}(\underline{a})$ is nilpotent. But that implies that $\underline{a} \in V\left(I_{\mathcal{A}}\right)$. Furthermore, since $M_{\mathcal{A}}(\underline{a})$ is a realization of $\mathcal{A}, a_{i_{k}, j_{k}} \neq 0$ for $k=1, \ldots, t$, or in other words, $\underline{a} \notin V\left(z_{i_{k}, j_{k}}\right)$ for each $k$. This proves the first direction.

For the reverse direction, if $\underline{a} \in V\left(I_{\mathcal{A}}\right) \backslash \bigcup_{k=1}^{t} V\left(z_{i_{k}, j_{k}}\right)$, then $M_{\mathcal{A}}(\underline{a})$ is a nilpotent matrix, and furthermore, since $a_{i_{k}, j_{k}} \neq 0$ for all $k$, this matrix is also a realization of $\mathcal{A}$.

As a consequence of Theorem 3.2, to determine if $\mathcal{A}$ is potentially nilpotent over $\mathbb{F}$, it suffices to show that the set $V\left(I_{\mathcal{A}}\right) \backslash \bigcup_{k=1}^{t} V\left(z_{i_{k}, j_{k}}\right)$ is non-empty. Unfortunately, this can be a highly non-trivial problem. However we can use this reformulation to describe an algebraic method to determine if the set $V\left(I_{\mathcal{A}}\right) \backslash \bigcup_{k=1}^{t} V\left(z_{i_{k}, j_{k}}\right)$ is empty, thus providing a means to determine if $\mathcal{A}$ is not potentially nilpotent over $\mathbb{F}$.

We begin with a simple lemma. A monomial of $R_{\mathcal{A}}$ is any polynomial of the form $m=z_{i_{1}, j_{1}}^{b_{1}} z_{i_{2}, j_{2}}^{b_{2}} \cdots z_{i_{t}, j_{t}}^{b_{t}}$ with each $b_{i} \in \mathbb{Z}_{\geq 0}$.

Lemma 3.3. Fix a field $\mathbb{F}$ and a znz-pattern $\mathcal{A}$. Suppose that there exists a monomial $m=z_{i_{1}, j_{1}}^{b_{1}} z_{i_{2}, j_{2}}^{b_{2}} \cdots z_{i_{t}, j_{t}}^{b_{t}} \in I_{\mathcal{A}}$. Then $\mathcal{A}$ is not potentially nilpotent over $\mathbb{F}$.

Proof. For any $\underline{a} \in V\left(I_{\mathcal{A}}\right)$, we must have $m(\underline{a})=a_{i_{1}, j_{1}}^{b_{1}} \cdots a_{i_{t}, j_{t}}^{b_{t}}=0$ because $m \in I_{\mathcal{A}}$. But this means that $a_{i_{k}, j_{k}}=0$ for some $k=1, \ldots, n$, and thus, $\underline{a} \in V\left(z_{i_{k}, j_{k}}\right)$. Now apply Theorem 3.2.

The colon operation and the saturation of ideals are two required algebraic ingredients:

Definition 3.4. Let $I$ and $J$ be ideals of a ring $R$. Then $I: J$ denotes the ideal

$$
I: J=\{g \in R \mid g J \subseteq I\} .
$$

The saturation of $I$ with respect to $J$, denoted $I: J^{\infty}$, is the ideal

$$
I: J^{\infty}=\left\{g \in R \mid g J^{i} \subseteq I \text { for some integer } i \geq 0\right\} .
$$

Alternatively, $I: J^{\infty}=(\cdots(((I: J): J): J) \cdots)$.

We come to one of the main results of this section. 
Theorem 3.5. Fix a field $\mathbb{F}$ and a znz-pattern $\mathcal{A}$. If $R_{\mathcal{A}}=\mathbb{F}\left[z_{i_{1}, j_{1}}, \ldots, z_{i_{t}, j_{t}}\right]$, then let $m_{\mathcal{A}}:=\prod_{k=1}^{t} z_{i_{k}, j_{k}}$ and let $J=\left(m_{\mathcal{A}}\right)$ be the ideal generated by $m_{\mathcal{A}}$. Then

(a) $V\left(I_{\mathcal{A}}\right) \backslash \bigcup_{k=1}^{t} V\left(z_{i_{k}, j_{k}}\right) \subseteq V\left(I_{\mathcal{A}}: J^{\infty}\right) \subseteq V\left(I_{\mathcal{A}}: J\right)$;

(b) if $1 \in I_{\mathcal{A}}: J^{\infty}$, then $\mathcal{A}$ is not potentially nilpotent over $\mathbb{F}$, or any field extension of $\mathbb{F}$;

(c) if $1 \in I_{\mathcal{A}}: J$, then $\mathcal{A}$ is not potentially nilpotent over $\mathbb{F}$, or any field extension of $\mathbb{F}$.

Proof. Statement $(a)$ is a well-known result via the algebraic geometry dictionary. For completeness, we include a short proof in this context. Suppose that $\underline{a} \in V\left(I_{\mathcal{A}}\right) \backslash$ $\bigcup_{k=1}^{t} V\left(z_{i_{k}, j_{k}}\right)$, and thus, $a_{i_{k}, j_{k}} \neq 0$ for $k=1, \ldots, t$. Suppose that $G \in I_{\mathcal{A}}: J^{\infty}$. Thus, there exists an integer $i$ such that $G J^{i} \subseteq I_{\mathcal{A}}$. But because $J^{i}=\left(m_{\mathcal{A}}^{i}\right)$, this implies that $G m_{\mathcal{A}}^{i} \in I_{\mathcal{A}}$. Since $\underline{a} \in V\left(I_{\mathcal{A}}\right)$, we have $\left(G m_{\mathcal{A}}^{i}\right)(\underline{a})=G(\underline{a}) m_{\mathcal{A}}^{i}(\underline{a})=0$. But since each $a_{i_{k}, j_{k}} \neq 0$, we have $m_{\mathcal{A}}^{i}(\underline{a})=a_{i_{1}, j_{1}}^{i} \cdots a_{i_{t}, j_{t}}^{i} \neq 0$, and hence $G(\underline{a})=0$, or equivalently, $\underline{a} \in V\left(I_{\mathcal{A}}: J^{\infty}\right)$. The second inclusion containment follows from the fact that $I_{\mathcal{A}}: J \subseteq I_{\mathcal{A}}: J^{\infty}$.

To prove $(b)$, suppose that $1 \in I_{\mathcal{A}}: J^{\infty}$. It then follows that there exists an $i$ such that $J^{i} \subseteq I_{\mathcal{A}}$, and hence $m_{\mathcal{A}}^{i} \in I_{\mathcal{A}}$. But then we get the desired conclusion by Lemma 3.3. In any extension of $\mathbb{F}$, we will continue to have $m_{\mathcal{A}}^{i} \in I_{\mathcal{A}}$. Statement $(c)$ follows directly from $(b)$ since we will have $1 \in I_{\mathcal{A}}: J \subseteq I_{\mathcal{A}}: J^{\infty}$.

REMARK 3.6. Many computer algebra systems allow one to compute the saturation of an ideal, thus making Theorem 3.5 a practical tool. The computational commutative algebra programs CoCoA [4] and Macaulay 2 [12] are two free programs that can be used to compute the ideals $I: J$ and $I: J^{\infty}$. On the second author's web page $^{2}$ is a short introduction on how to use these programs to compute the examples found below.

Some well-known necessary facts for nilpotent matrices are simple corollaries of Theorem 3.5.

Corollary 3.7. Let $\mathcal{A}$ be znz-pattern. If $\mathcal{A}$ has only one nonzero entry on the diagonal or only one transversal, then $\mathcal{A}$ is not potentially nilpotent over any field $\mathbb{F}$.

Proof. In both cases, we show that one of the generators of $I_{\mathcal{A}}$ must be a monomial. If $\mathcal{A}$ has only one nonzero entry on the diagonal, say at position $(i, i)$, then the trace of $M_{\mathcal{A}}$ is $z_{i, i}$. But since $F_{1}=\operatorname{tr} M_{\mathcal{A}}=z_{i, i}$, it immediately follows that $m_{\mathcal{A}} \in I_{\mathcal{A}}$, and hence, $1 \in I_{\mathcal{A}}:\left(m_{\mathcal{A}}\right)$. Similarly, if $\mathcal{A}$ has only one transversal, the determinant of $M_{\mathcal{A}}$, which equals $F_{n}$, has form $z_{i_{1}, j_{1}}^{b_{1}} z_{i_{2}, j_{2}}^{b_{2}} \cdots z_{i_{t}, j_{t}}^{b_{t}}$ where $b_{k}=1$ or 0 . It then follows that $m_{\mathcal{A}} \in I_{\mathcal{A}}$, or equivalently, $1 \in I_{\mathcal{A}}:\left(m_{\mathcal{A}}\right)$.

\footnotetext{
${ }^{2}$ http://flash.lakeheadu.ca/ avantuyl/research/research.html
} 
We now provide some illustrative examples.

ExAmple 3.8. Let $\mathcal{A}$ be the znz-pattern of Example 3.1. Let $\mathbb{F}$ be any field of characteristic two. Because

$$
I_{\mathcal{A}}=\left(z_{1,1}+z_{3,3}, \quad z_{1,2} z_{2,1}+z_{2,3} z_{3,2}+z_{1,1} z_{3,3}, \quad z_{1,1} z_{2,3} z_{3,2}+z_{1,2} z_{2,1} z_{3,3}\right),
$$

the monomial $z_{1,1}^{2} z_{3,3} \in I_{\mathcal{A}}$ because

$$
\begin{gathered}
z_{1,2} z_{2,1}\left(z_{1,1}+z_{3,3}\right)+z_{1,1}\left(z_{1,2} z_{2,1}+z_{2,3} z_{3,2}+z_{1,1} z_{3,3}\right)+\left(z_{1,1} z_{2,3} z_{3,2}+z_{1,2} z_{2,1} z_{3,3}\right) \\
=2 z_{1,2} z_{2,1} z_{1,1}+2 z_{1,1} z_{2,3} z_{3,2}+z_{1,1}^{2} z_{3,3}+2 z_{1,2} z_{2,1} z_{3,3}=z_{1,1}^{2} z_{3,3}
\end{gathered}
$$

since $x+x=0$ for any $x \in \mathbb{F}$. Thus $\mathcal{A}$ is not potentially nilpotent over any field of extension of $\mathbb{F}$. Note that when $\mathbb{F}=\mathbb{Z}_{2}$ is the finite field with exactly two elements, then one could use a direct calculation because there is only one choice for each $z_{i, j}$, namely $1_{\mathbb{F}}$. However, this method shows that $\mathcal{A}$ is not potentially nilpotent over any extension of this field.

ExAmple 3.9. It is possible that $1 \in I_{\mathcal{A}}: J^{\infty}$, but $1 \notin I_{\mathcal{A}}: J$. As an example, consider the znz-pattern

$$
\mathcal{A}=\left[\begin{array}{lll}
* & 0 & 0 \\
0 & * & * \\
0 & * & *
\end{array}\right]
$$

We can see immediately that $\mathcal{A}$ is not potentially nilpotent over any field $\mathbb{F}$ since any realization $A$ of $\mathcal{A}$ will have the nonzero eigenvalue of $a_{1,1}$. However, this cannot be deduced from $I_{\mathcal{A}}: J$. For example, if $\mathbb{F}=\mathbb{Z}_{2}$, then we use CoCoA or Macaulay 2 to find $I_{\mathcal{A}}: J=$

$$
\begin{aligned}
& \left(z_{1,1}+z_{2,2}+z_{3,3},-z_{1,1} z_{2,2}+z_{2,3} z_{3,2}-z_{1,1} z_{3,3}-z_{2,2} z_{3,3},-z_{1,1} z_{2,3} z_{3,2}+z_{1,1} z_{2,2} z_{3,3}\right):\left(m_{\mathcal{A}}\right) \\
& =\left(z_{1,1}+z_{2,2}+z_{3,3}, z_{2,2}^{2}+z_{3,3}^{2}, z_{2,3} z_{3,2}+z_{2,2} z_{3,3}\right) .
\end{aligned}
$$

However, a computer algebra system will reveal that $1 \in I_{\mathcal{A}}: J^{\infty}$, thus showing that $\mathcal{A}$ is not potentially nilpotent over $\mathbb{F}$.

EXAMPLE 3.10. Using the saturation of ideals also lends itself to sign patterns. Consider the signed pattern

$$
\mathcal{A}=\left[\begin{array}{ccccc}
- & - & - & 0 & 0 \\
+ & + & + & 0 & 0 \\
0 & 0 & 0 & - & - \\
0 & - & 0 & 0 & - \\
- & 0 & 0 & 0 & 0
\end{array}\right] .
$$


The pattern $\mathcal{A}$ is the pattern $\mathcal{G}_{5}$ studied in [14]. We then consider the matrix

$$
M_{\mathcal{A}}=\left[\begin{array}{ccccc}
-z_{1,1} & -z_{1,2} & -z_{1,3} & 0 & 0 \\
z_{2,1} & z_{2,2} & z_{2,3} & 0 & 0 \\
0 & 0 & 0 & -z_{3,4} & -z_{3,5} \\
0 & -z_{4,2} & 0 & 0 & -z_{4,5} \\
-z_{5,1} & 0 & 0 & 0 & 0
\end{array}\right] .
$$

We define $I_{\mathcal{A}}$ as above. Letting $\mathbb{F}=\mathbb{R}$, we find that $1 \in I_{\mathcal{A}}:\left(m_{\mathcal{A}}\right)^{\infty}$ using CoCoA. This sign pattern $\mathcal{A}$, therefore, is not potentially nilpotent over $\mathbb{R}$, as first discovered in [14]; in fact $\mathcal{G}_{5}$ is part of a much larger family of non-potentially nilpotent patterns.

As we will show below, the converse of Theorem 3.5 (b) does not hold. To show that $\mathcal{A}$ is not potentially nilpotent, we apply the theory of Gröbner bases. Roughly speaking, a Gröbner basis of $I_{\mathcal{A}}$ is a "good" choice of generators of $I_{\mathcal{A}}$ which can allow one to describe the affine variety $V\left(I_{\mathcal{A}}\right)$.

We now recall the needed definitions. We fix a monomial ordering $>$ on the monomials of $R_{\mathcal{A}}$, that is, (1) > is a total ordering on the set of monomials, (2) > is compatible with multiplication (if $m_{1}>m_{2}$, then for any monomial $m, m m_{1}>m m_{2}$ ), and $(3)>$ is also a well-ordering. Of particular importance is the lex monomial ordering, that is,

$$
z_{i_{1}, j_{1}}^{a_{1}} z_{i_{2}, j_{2}}^{a_{2}} \cdots z_{i_{t}, j_{t}}^{a_{t}}>z_{i_{1}, j_{1}}^{b_{1}} z_{i_{2}, j_{2}}^{b_{2}} \cdots z_{i_{t}, j_{t}}^{b_{t}}
$$

if and only if the first nonzero entry of the $t$-tuple $\left(a_{1}-b_{1}, \ldots, a_{t}-b_{t}\right)$ is positive.

For any polynomial $F=\sum c_{\alpha} m_{\alpha} \in R_{\mathcal{A}}$ where $m_{\alpha}$ are monomials and $c_{\alpha} \in \mathbb{F}$, the leading term of $F$, denoted $\operatorname{LT}_{>}(F)$ is the largest monomial term $c_{\alpha} m_{\alpha}$ in $F$ with respect to $>$.

Definition 3.11. A subset $\left\{G_{1}, \ldots, G_{s}\right\}$ of an ideal $I$ is a Gröbner basis of $I$ with respect to a monomial ordering $>$ if for all $F \in I, L T_{>}(F)$ is divisible by $L T_{>}\left(G_{i}\right)$ for some $i$.

We then make use of the following two properties of Gröbner bases.

Theorem 3.12. Let $R=\mathbb{F}\left[z_{i_{1}, j_{1}}, \ldots, z_{i_{t}, j_{t}}\right]$. Let $>$ be the lex monomial ordering with the property that $z_{i_{1}, j_{1}}>\cdots>z_{i_{t}, j_{t}}$. Let $I$ be an ideal of $R$, and suppose that $\left\{G_{1}, \ldots, G_{s}\right\}$ is a Gröbner basis of I with respect to $>$. Then

(a) $I=\left(G_{1}, \ldots, G_{s}\right)$, that is, the Gröbner basis generates $I$;

(b) Let $I_{l}=I \cap \mathbb{F}\left[z_{i_{l+1}, j_{l+1}}, \ldots, z_{i_{t}, j_{t}}\right]$. Then $I_{l}$ is the lth elimination ideal, and a Gröbner basis for $I_{l}$ is $\left\{G_{1}, \ldots, G_{s}\right\} \cap \mathbb{F}\left[z_{i_{l+1}, j_{l+1}}, \ldots, z_{i_{t}, j_{t}}\right]$.

Proof. Statement (a) is [6, Chapter 2, $\S 5$, Corollary 2], while (b) is known as the Elimination Theorem [6, Chapter $3, \S 1$, Theorem 2]. 
To make use of the above theorem to describe the affine variety $V(I)$, one first finds a Gröbner basis $\left\{G_{1}, \ldots, G_{s}\right\}$ for $I$ with respect to the lex monomial order. Theorem 3.12(b) implies that we can partition the $G_{i}$ 's so that the first set are polynomials in the variables $\left\{z_{i_{1}, j_{1}}, \ldots, z_{i_{t}, j_{t}}\right\}$, the second set are polynomials in the variables $\left\{z_{i_{2}, j_{2}}, \ldots, z_{i_{t}, j_{t}}\right\}$, and so on, i.e., the number of variables is eliminated as you move through the partitions. In some (but not all) cases, one or more of the $G_{i}$ 's may only contain one variable. We can then find roots of these polynomials (either explicitly or numerically), and then using these solutions, find roots to the other polynomials.

We illustrate how to use Gröbner bases to eliminate some znz-patterns $\mathcal{A}$ as being potentially nilpotent over $\mathbb{F}$. We will study the following pattern in more detail in the next section.

EXAMPLE 3.13. We consider the znz-pattern

$$
\mathcal{A}=\left[\begin{array}{lll}
* & * & 0 \\
0 & * & * \\
* & 0 & *
\end{array}\right]
$$

and let $\mathbb{F}=\mathbb{R}$. In this case, the generators of the ideal $I_{\mathcal{A}}$ are

$$
I_{\mathcal{A}}=\left(z_{1,1}+z_{2,2}+z_{3,3}, \quad z_{1,1} z_{2,2}+z_{1,1} z_{3,3}+z_{2,2} z_{3,3}, \quad z_{1,2} z_{2,3} z_{3,1}+z_{, 1} z_{2,2} z_{3,3}\right) .
$$

We can use a computer algebra program to check that $I_{\mathcal{A}}:\left(z_{1,1} z_{1,2} z_{2,2} z_{2,3} z_{3,1} z_{3,3}\right)^{\infty} \neq$ (1). Thus Theorem 3.5 does not tell us if $\mathcal{A}$ is not potentially nilpotent over $\mathbb{R}$.

We use either CoCoA or Macaulay 2 to find a Gröbner basis for $I_{\mathcal{A}}$ :

$$
\left\{z_{1,1}+z_{2,2}+z_{3,3}, \quad z_{1,2} z_{2,3} z_{3,1}+z_{3,3}^{3}, \quad z_{2,2}^{2}+z_{2,2} z_{3,3}+z_{3,3}^{2}\right\} .
$$

Notice that the last polynomial contains the fewest number of variables. If $\mathcal{A}$ was potentially nilpotent, then there exists $\underline{a}=\left(a_{1,1}, a_{1,2}, a_{2,2}, a_{2,3}, a_{3,1}, a_{3,3}\right) \in \mathbb{R}^{6}$ such that $M_{\mathcal{A}}(\underline{a})$ is nilpotent, and specifically, $\underline{a}$ is a zero of all three polynomials in the Gröbner basis. Note $a_{3,3}$ must be a nonzero real number. But for any nonzero real number $a \in \mathbb{R}$, the last polynomial from the Gröbner basis implies that $a_{2,2}$ will then have to satisfy

$$
z_{2,2}^{2}+a z_{2,2}+a^{2}=0 \Leftrightarrow z_{2,2}=a\left(\frac{-1 \pm \sqrt{-3}}{2}\right) .
$$

But then for every nonzero choice of $a \in \mathbb{R}, a_{2,2} \notin \mathbb{R}$. Hence, $\mathcal{A}$ is not potentially nilpotent over $\mathbb{R}$. Observe that this example shows that the converse of Theorem 3.5(b) is false.

4. Digraphs without $k$-cycles with $k$ small: a necessary condition. Let $D(\mathcal{A})$ be the digraph associated to a znz-pattern $\mathcal{A}$. It is known that if $\mathcal{A}$ is potentially 
nilpotent over $\mathbb{F}=\mathbb{R}$, and if $D(\mathcal{A})$ has at least two loops, then $D(\mathcal{A})$ must have a 2-cycle. See, for example, [7, Lemma 3.2] which considers the signed case, but the proof also holds in the non-signed case. When $\mathbb{F} \neq \mathbb{R}$, then this necessary condition need not hold:

EXAmple 4.1. Let $\mathcal{A}$ be the pattern of Example 3.13. The associated graph $D(\mathcal{A})$ has three loops but no two cycles, so [7, Lemma 3.2] implies that $\mathcal{A}$ is not potentially nilpotent over $\mathbb{R}$. However, $\mathcal{A}$ is potentially nilpotent over other fields: Bodine [1] has observed that $\mathcal{A}$ is SAP (hence potentially nilpotent) over certain finite fields and Yielding [21] has observed that $\mathcal{A}$ is SAP over $\mathbb{C}$. For example, $\mathcal{A}$ is potentially nilpotent over $\mathbb{F}=\mathbb{Z}_{7}$ as demonstrated with the realization

$$
\left[\begin{array}{ccc}
4_{\mathbb{F}} & 1_{\mathbb{F}} & 0 \\
0 & 2_{\mathbb{F}} & 1_{\mathbb{F}} \\
-1_{\mathbb{F}} & 0 & 1_{\mathbb{F}}
\end{array}\right] .
$$

Our goal in this section is to explore the role of small cycles in nilpotence. More precisely, we provide a necessary condition on $\mathbb{F}$ for a znz-pattern $\mathcal{A}$ to be potentially nilpotent over $\mathbb{F}$ if $D(\mathcal{A})$ has loops, but no $k$-cycles of small size. We begin by recalling the definition of the roots of unity and one result concerning these numbers.

Definition 4.2. Fix a field $\mathbb{F}$. We say that $\mathbb{F}$ contains all the $m^{\text {th }}$ roots of unity if all of the $m$ roots of the polynomial $x^{m}-1_{\mathbb{F}}=\left(x-1_{\mathbb{F}}\right)\left(x^{m-1}+x^{m-2}+\cdots+x+1_{\mathbb{F}}\right)$ belong to $\mathbb{F}$, that is, $x^{m}-1_{\mathbb{F}}$ factors into $m$ linear forms over $\mathbb{F}$.

Lemma 4.3. Fix a field $\mathbb{F}$ and integer $m \geq 2$. Suppose that there is a solution $\left(a_{1}, \ldots, a_{m}\right) \in \mathbb{F}^{m}$ to the $m-1$ elementary symmetric polynomial equations

$$
\begin{aligned}
z_{1}+z_{2}+\cdots+z_{m} & =0 \\
z_{1} z_{2}+\cdots+z_{m-1} z_{m} & =0 \\
& \vdots \\
z_{1} z_{2} \cdots z_{m-1}+\cdots+z_{2} z_{3} \cdots z_{m} & =0
\end{aligned}
$$

with all $a_{j} \neq 0$. Then $\mathbb{F}$ contains all the $m^{\text {th }}$ roots of unity.

Proof. If $\left(a_{1}, \ldots, a_{m}\right)$ is such a solution, then $\left(a_{1} a_{m}^{-1}, \ldots, a_{m} a_{m}^{-1}\right)$ is also a solution. Thus, we can assume $a_{m}=1_{\mathbb{F}}$. Hence, substituting $\left(a_{1}, \ldots, a_{m-1}, 1_{\mathbb{F}}\right)$ into the above equations and rearranging gives:

$$
\begin{aligned}
a_{1}+a_{2}+\cdots+a_{m-1} & =-1_{\mathbb{F}} \\
a_{1} a_{2}+\cdots+a_{m-2} a_{m-1} & =1_{\mathbb{F}} \\
& \vdots \\
a_{1} a_{2} \cdots a_{m-1} & =\left(-1_{\mathbb{F}}\right)^{m-1} .
\end{aligned}
$$


We claim that $a_{1}, \ldots, a_{m-1}$ are the remaining $m^{\text {th }}$ roots of unity. Indeed,

$$
\begin{aligned}
\left(x-a_{1}\right)\left(x-a_{2}\right) \cdots\left(x-a_{m-1}\right)= & x^{m-1}-\left(a_{1}+a_{2}+\cdots a_{m-1}\right) x^{m-2} \\
& +\left(a_{1} a_{2}+\cdots+a_{m-2} a_{m-1}\right) x^{m-3} \\
& +\cdots+(-1)^{m-2}\left(a_{1} \cdots a_{m-2}+\cdots+a_{2} \cdots a_{m-1}\right) x \\
& +(-1)^{m-1} a_{1} \cdots a_{m-1} \\
= & x^{m-1}+x^{m-2}+\cdots+x+1_{\mathbb{F}} .
\end{aligned}
$$

That is, $a_{1}, \ldots, a_{m-1}$ are the zeros of $x^{m-1}+x^{m-2}+\cdots+x+1_{\mathbb{F}}$. The conclusion now follows.

TheOREM 4.4. Let $\mathcal{A}$ be a znz-pattern with $m \geq 2$ nonzero entries on the diagonal, and suppose that $D(\mathcal{A})$ has no $k$-cycles with $2 \leq k \leq m-1$. If $\mathcal{A}$ is potentially nilpotent over $\mathbb{F}$, then $\mathbb{F}$ contains all the $m^{\text {th }}$ roots of unity.

Proof. After relabeling, we may assume that the nonzero diagonal entries of $\mathcal{A}$ are at $(1,1), \ldots,(m, m)$. To simplify notation, let $z_{i}$ denote the variable $z_{i, i}$ in the polynomial ring $R_{\mathcal{A}}$. Because $D(\mathcal{A})$ has no $k$-cycles with $2 \leq k \leq m-1$, this implies that the first $m-1$ generators of $I_{\mathcal{A}}$ are:

$$
\begin{aligned}
F_{1} & =z_{1}+z_{2}+\cdots+z_{m} \\
F_{2} & =z_{1} z_{2}+\cdots+z_{m-1} z_{m} \\
& \vdots \\
F_{m-1} & =z_{1} z_{2} \cdots z_{m-1}+\cdots+z_{2} z_{3} \cdots z_{m} .
\end{aligned}
$$

Let $A \in Q(\mathcal{A})$ be a realization that is nilpotent. If $a_{1,1}, \ldots, a_{m, m}$ are the nonzero diagonal entries, then $\underline{a}=\left(a_{1,1}, \ldots, a_{m, m}\right)$ satisfies $F_{i}(\underline{a})=0$ for $i=1, \ldots, m-1$. Because $a_{j, j} \neq 0$ for $1 \leq j \leq m$, Lemma 4.3 implies that the field $\mathbb{F}$ contains all the $m^{\text {th }}$ roots of unity.

Corollary 4.5. Let $\mathcal{A}$ be a znz-pattern with $m \geq 2$ nonzero entries on the diagonal, and suppose that $D(\mathcal{A})$ has no $k$-cycles with $2 \leq k \leq m$. Then $\mathcal{A}$ is not potentially nilpotent over any $\mathbb{F}$.

Proof. We use the notation of the proof of Theorem 4.4. Because $\mathcal{A}$ has no $k$-cycle with $2 \leq k \leq m$, we have $F_{m}=z_{1} z_{2} \cdots z_{m} \in I_{\mathcal{A}}$. Now apply Lemma 3.3.

Using the above theorem, we can give a infinite family $\mathcal{A}_{n}$ below of potentially nilpotent znz-patterns. In [2], this family was demonstrated to fail to be potentially nilpotent for $\mathbb{F}=\mathbb{R}$.

Theorem 4.6. Fix a field $\mathbb{F}$, and for each $n \geq 3$, let $\mathcal{A}_{n}$ denote the $n \times n$ 
znz-pattern

$$
\mathcal{A}_{n}=\left[\begin{array}{cccccc}
* & * & 0 & \cdots & \cdots & 0 \\
0 & * & * & \ddots & & \vdots \\
\vdots & \ddots & \ddots & \ddots & \ddots & \vdots \\
\vdots & & & \ddots & * & 0 \\
0 & 0 & & \ddots & * & * \\
* & 0 & 0 & \cdots & 0 & *
\end{array}\right]
$$

Then $\mathcal{A}_{n}$ is potentially nilpotent over $\mathbb{F}$ if and only if $\mathbb{F}$ contains all the $n^{\text {th }}$ roots of unity.

Proof. The graph of $D(\mathcal{A})$ is an $n$-cycle with a loop at each vertex. Thus, one direction follows immediately from Theorem 4.4 since $D(\mathcal{A})$ has $n$ loops and no $k$ cycles for $2 \leq k \leq n-1$. For the converse direction, suppose that $\mathbb{F}$ contains all the $n^{\text {th }}$ roots of unity: suppose $x^{n}-1_{\mathbb{F}}=\left(x-\zeta_{1}\right) \cdots\left(x-\zeta_{n-1}\right)\left(x-1_{\mathbb{F}}\right)$ with $\zeta_{1}, \ldots, \zeta_{n-1} \in \mathbb{F}$. Then the matrix

$$
A_{n}=\left[\begin{array}{cccccc}
\zeta_{1} & 1_{\mathbb{F}} & 0 & \cdots & \cdots & 0 \\
0 & \zeta_{2} & 1_{\mathbb{F}} & 0 & & 0 \\
\vdots & \ddots & \ddots & \ddots & \ddots & \vdots \\
\vdots & & & \ddots & \ddots & 0 \\
0 & 0 & & \ddots & \zeta_{n-1} & 1_{\mathbb{F}} \\
-1_{\mathbb{F}} & 0 & 0 & \cdots & 0 & 1_{\mathbb{F}}
\end{array}\right]
$$

is a desired realization.

Corollary 4.7. Fix a prime $p$. If $p \equiv 1(\bmod n)$, then $\mathcal{A}_{n}$ is potentially nilpotent over $\mathbb{F}=\mathbb{Z}_{p}$.

Proof. When $p \equiv 1(\bmod n)$, then by $\left[16\right.$, Theorem 2.4], the field $\mathbb{Z}_{p}$ contains all the $n^{\text {th }}$ roots of unity. Now apply the Theorem 4.6.

EXAMPLE 4.8. Theorem 4.6 gives a new way to explain why the pattern $\mathcal{A}=\mathcal{A}_{3}$ of Example 3.13 is not potentially nilpotent over $\mathbb{R}$. Because $D(\mathcal{A})$ has three loops, but no two cycles, if $\mathcal{A}$ were potentially nilpotent over $\mathbb{F}$, then $\mathbb{F}$ must contain all the cube roots of unity. However, $\mathbb{R}$ does not have this property. But when $\mathbb{F}=\mathbb{Z}_{7}$, $x^{3}-1$ factors into linear forms; hence the realization in Example 4.1.

5. Potentially nilpotent matrices of small order over finite fields. In this section, we employ the tools of previous sections to classify all znz-patterns $\mathcal{A}$ 
that are potentially nilpotent over $\mathbb{F}$ of order two or three when $\mathbb{F}=\mathbb{Z}_{p}$, with $p$ a prime number. As a consequence of Lemma 2.1, it suffices to classify all znz-patterns of order two or three that are irreducible.

We begin with the $2 \times 2$ case by showing a much stronger result:

TheOREm 5.1. Let $\mathbb{F}$ be any field. Then the znz-pattern

$$
\mathcal{A}=\left[\begin{array}{ll}
* & * \\
* & *
\end{array}\right]
$$

is the only irreducible $2 \times 2$ potentially nilpotent pattern over $\mathbb{F}$.

Proof. The only irreducible $2 \times 2$ znz-patterns are

$$
\left[\begin{array}{ll}
0 & * \\
* & 0
\end{array}\right],\left[\begin{array}{cc}
* & * \\
* & 0
\end{array}\right],\left[\begin{array}{ll}
0 & * \\
* & *
\end{array}\right] \text {, and }\left[\begin{array}{ll}
* & * \\
* & *
\end{array}\right] \text {. }
$$

The first three patterns cannot be potentially nilpotent over $\mathbb{F}$ by Corollary 3.7. The matrix

$$
\left[\begin{array}{cc}
1_{\mathbb{F}} & 1_{\mathbb{F}} \\
-1_{\mathbb{F}} & -1_{\mathbb{F}}
\end{array}\right]
$$

is a desired realization of $\mathcal{A}$.

The following lemma is used to shorten some of the cases in the next theorem.

Lemma 5.2. Let $\mathcal{A}$ be an irreducible $n \times n$ znz-pattern. Let $D(\mathcal{A})$ be the associated digraph.

(a) If $\mathbb{F}=\mathbb{Z}_{2}$ and $D(\mathcal{A})$ has an odd number of loops, then $\mathcal{A}$ is not potentially nilpotent over $\mathbb{F}$.

(b) If $\mathbb{F}=\mathbb{Z}_{2}$ and $D(\mathcal{A})$ has exactly two loops and two 2-cycles, then $\mathcal{A}$ is not potentially nilpotent over $\mathbb{F}=\mathbb{Z}_{2}$.

(c) The only solutions to the equation $x+y+z=0$ with $x, y, z \in \mathbb{Z}_{3}$ and $x, y$, $z$ nonzero are $\left(1_{\mathbb{F}}, 1_{\mathbb{F}}, 1_{\mathbb{F}}\right)$ and $\left(2_{\mathbb{F}}, 2_{\mathbb{F}}, 2_{\mathbb{F}}\right)$.

Proof. (a) Suppose that $D(\mathcal{A})$ has loops at $\left(i_{1}, i_{1}\right), \ldots,\left(i_{m}, i_{m}\right)$ with $m=2 k+1$. Then $z_{i_{1}, i_{1}}+\cdots+z_{i_{m}, i_{m}} \in I_{\mathcal{A}}$. When $\mathbb{F}=\mathbb{Z}_{2}$, we must have $z_{i, j}=1_{\mathbb{F}}$ for all $(i, j)$. But this would imply that $1_{\mathbb{F}}+\cdots+1_{\mathbb{F}}=m_{\mathbb{F}}=0$, which is false.

(b) Suppose that the diagonal entries of $\mathcal{A}$ are at $\left(i_{1}, i_{1}\right)$ and $\left(i_{2}, i_{2}\right)$ and the two 2 -cycles are $\left(i_{3}, j_{3}\right)$ and $\left(i_{4}, j_{4}\right)$. Then the polynomial

$$
F_{2}=z_{i_{1}, i_{1}} z_{i_{2}, i_{2}}+z_{i_{3}, j_{3}} z_{j_{3}, i_{3}}+z_{i_{4}, j_{4}} z_{j_{4}, i_{4}} \in I_{\mathcal{A}} .
$$

If $\mathbb{F}=\mathbb{Z}_{2}$, then the only nonzero choice for $z_{i, j}$ is $1_{\mathbb{F}}$. It then follows that $F_{2}$ cannot equal zero in $\mathbb{F}=\mathbb{Z}_{2}$. 
(c) This statement follows from inspection.

We say that two patterns are equivalent if they have the same digraph.

Theorem 5.3. Fix a prime $p$ and an irreducible $3 \times 3$ znz-pattern $\mathcal{A}$. Then $\mathcal{A}$ is potentially nilpotent over $\mathbb{F}=\mathbb{Z}_{p}$ if and only if, up to equivalence, $\mathcal{A}$ and $p$ have one of the following forms:

1. $\mathcal{A}=\left[\begin{array}{lll}0 & * & 0 \\ * & 0 & * \\ 0 & * & 0\end{array}\right]\left[\begin{array}{ccc}* & * & 0 \\ * & 0 & * \\ * & 0 & *\end{array}\right]\left[\begin{array}{lll}0 & * & 0 \\ * & * & * \\ * & 0 & *\end{array}\right]$ or $\left[\begin{array}{ccc}* & * & * \\ * & * & * \\ * & * & 0\end{array}\right]$, and p arbitrary.

2. $\mathcal{A}=\left[\begin{array}{lll}* & * & 0 \\ * & 0 & * \\ 0 & * & *\end{array}\right]\left[\begin{array}{lll}* & * & * \\ * & * & * \\ * & 0 & 0\end{array}\right]\left[\begin{array}{ccc}* & * & * \\ * & 0 & * \\ * & 0 & *\end{array}\right]\left[\begin{array}{lll}* & * & * \\ * & * & * \\ * & 0 & *\end{array}\right]\left[\begin{array}{lll}0 & * & * \\ * & 0 & * \\ * & * & 0\end{array}\right]$ or $\left[\begin{array}{lll}* & * & * \\ * & * & * \\ * & * & *\end{array}\right]$,

and $p \neq 2$.

3. $\mathcal{A}=\left[\begin{array}{lll}* & * & 0 \\ * & * & * \\ 0 & * & *\end{array}\right]\left[\begin{array}{lll}* & * & 0 \\ * & * & * \\ * & 0 & *\end{array}\right]$ or $\left[\begin{array}{lll}0 & * & * \\ * & * & * \\ * & 0 & *\end{array}\right]$, and $p \neq 2$ or 3 .

4. $\mathcal{A}=\left[\begin{array}{lll}* & * & 0 \\ 0 & * & * \\ * & 0 & *\end{array}\right]$, and $\mathbb{F}=\mathbb{Z}_{p}$ contains the cube roots of unity.

Proof. We do a case-by-case analysis, by considering all $3 \times 3$ irreducible znzpatterns $\mathcal{A}$. Recall that a pattern $\mathcal{A}$ is irreducible if and only if the digraph $D(\mathcal{A})$ is strongly connected. We break our proof into five cases, where each case corresponds to one of the five non-isomorphic graphs on three vertices that is strongly connected and with no loops. Each case is then broke into sub-cases, where each sub-case considers the locations of the loops.

Case 1. The non-loop edges are $(1,2),(2,3)$, and $(3,1)$.

In this case, we need to consider four znz-patterns:

$$
\mathcal{A}_{1,1}=\left[\begin{array}{lll}
0 & * & 0 \\
0 & 0 & * \\
* & 0 & 0
\end{array}\right] \quad \mathcal{A}_{1,2}=\left[\begin{array}{lll}
* & * & 0 \\
0 & 0 & * \\
* & 0 & 0
\end{array}\right] \quad \mathcal{A}_{1,3}=\left[\begin{array}{ccc}
* & * & 0 \\
0 & * & * \\
* & 0 & 0
\end{array}\right] \quad \mathcal{A}_{1,4}=\left[\begin{array}{ccc}
* & * & 0 \\
0 & * & * \\
* & 0 & *
\end{array}\right]
$$

Patterns $\mathcal{A}_{1,1}, \mathcal{A}_{1,2}$, and $\mathcal{A}_{1,3}$ cannot be potentially nilpotent over any field $\mathbb{F}$ by Corollary 3.7 since one of the generators of $I_{A_{1, j}}$ for $j=1,2,3$ will be a monomial. On the other hand, $\mathcal{A}_{1,4}$ is potentially nilpotent over $\mathbb{F}$ if and only if the polynomial $x^{3}-1_{\mathbb{F}}$ factors into linear forms in $\mathbb{F}=\mathbb{Z}_{p}$. This is a special case of Theorem 4.6.

Case 2: The non-loop edges are $(1,2),(2,1),(2,3)$, and $(3,2)$. 
In this case, we need to consider six znz-patterns:

$$
\begin{gathered}
\mathcal{A}_{2,1}=\left[\begin{array}{lll}
0 & * & 0 \\
* & 0 & * \\
0 & * & 0
\end{array}\right] \mathcal{A}_{2,2}=\left[\begin{array}{lll}
* & * & 0 \\
* & 0 & * \\
0 & * & 0
\end{array}\right] \mathcal{A}_{2,3}=\left[\begin{array}{lll}
0 & * & 0 \\
* & * & * \\
0 & * & 0
\end{array}\right] \\
\mathcal{A}_{2,4}=\left[\begin{array}{lll}
* & * & 0 \\
* & * & * \\
0 & * & 0
\end{array}\right] \mathcal{A}_{2,5}=\left[\begin{array}{lll}
* & * & 0 \\
* & 0 & * \\
0 & * & *
\end{array}\right] \mathcal{A}_{2,6}=\left[\begin{array}{lll}
* & * & 0 \\
* & * & * \\
0 & * & *
\end{array}\right] .
\end{gathered}
$$

We can eliminate patterns $\mathcal{A}_{2,2}, \mathcal{A}_{2,3}$ and $\mathcal{A}_{2,4}$ as being potentially nilpotent over any $\mathbb{F}$ by using Corollary 3.7. Pattern $\mathcal{A}_{2,1}$ is potentially nilpotent over any $\mathbb{F}$ since $1_{\mathbb{F}},-1_{\mathbb{F}} \in \mathbb{F}$ and

$$
\left[\begin{array}{ccc}
0 & 1_{\mathbb{F}} & 0 \\
1_{\mathbb{F}} & 0 & 1_{\mathbb{F}} \\
0 & -1_{\mathbb{F}} & 0
\end{array}\right]
$$

is a desired realization.

Using Theorem 3.5 and CoCoA, we can show that the pattern ${ }^{3} \mathcal{A}_{2,5}$ is not potentially nilpotent over $\mathbb{F}=\mathbb{Z}_{2}$ (or alternatively, see Examples 3.1 and 3.8). If $p \neq 2$, then $\mathcal{A}$ is potentially nilpotent over $\mathbb{F}=\mathbb{Z}_{p}$ since

$$
\left[\begin{array}{ccc}
1_{\mathbb{F}} & -2_{\mathbb{F}}^{-1} & 0 \\
1_{\mathbb{F}} & 0 & 2_{\mathbb{F}}^{-1} \\
0 & -1_{\mathbb{F}} & -1_{\mathbb{F}}
\end{array}\right]
$$

is a desired realization.

The pattern $\mathcal{A}_{2,6}$ is not potentially nilpotent over $\mathbb{F}=\mathbb{Z}_{2}$ by Lemma 5.2 (a). Also, $\mathcal{A}_{2,6}$ is not potentially nilpotent over $\mathbb{F}=\mathbb{Z}_{3}$. We can show this by calculating the Gröbner basis of $I_{\mathcal{A}_{2,6}}$ in the ring $R=\mathbb{Z}_{3}\left[z_{1,1}, z_{1,2}, z_{2,1}, z_{2,2}, z_{2,3}, z_{3,2}, z_{3,3}\right]$ :

$$
\left\{z_{1,1}+z_{2,2}+z_{3,3}, \quad z_{1,2} z_{2,1}+z_{2,2}^{2}+z_{2,3} z_{3,2}+z_{2,2} z_{3,3}+z_{3,3}^{2}, \quad z_{2,2} z_{2,3} z_{3,2}-z_{2,3} z_{3,2} z_{3,3}+z_{3,3}^{3}\right\} .
$$

Since $z_{1,1}+z_{2,2}+z_{3,3}=0$, by Lemma 5.2 (c), we need only consider the cases that $z_{1,1}=z_{2,2}=z_{3,3}=1_{\mathbb{F}}$ or they all equal $2_{\mathbb{F}}$. In either case, solving for nonzero roots of the last polynomial of the Gröbner basis we get $z_{2,3} z_{3,2}-z_{2,3} z_{3,2}+1_{\mathbb{F}}=0$ or $2_{\mathbb{F}} z_{2,3} z_{3,2}-2_{\mathbb{F}} z_{2,3} z_{3,2}+2_{\mathbb{F}}=0$, neither of which has a solution in $\mathbb{Z}_{3}$. So, this pattern is not potentially nilpotent of $\mathbb{Z}_{3}$. On the other hand, if $p \neq 3$, then

$$
\left[\begin{array}{ccc}
2_{\mathbb{F}} & 2_{\mathbb{F}} & 0 \\
-4_{\mathbb{F}} & -3_{\mathbb{F}} & 1_{\mathbb{F}} \\
0 & 1_{\mathbb{F}} & 1_{\mathbb{F}}
\end{array}\right]
$$

\footnotetext{
${ }^{3}$ The pattern $\mathcal{A}_{2,5}$ is the antipodal tridiagonal pattern $T_{3}$ studied in [8].
} 
is a desired realization.

Case 3. The non-loop edges are $(1,2),(2,1),(2,3)$, and $(3,1)$.

We now need to consider eight znz-patterns:

$$
\begin{aligned}
& \mathcal{A}_{3,1}=\left[\begin{array}{lll}
0 & * & 0 \\
* & 0 & * \\
* & 0 & 0
\end{array}\right] \quad \mathcal{A}_{3,2}=\left[\begin{array}{ccc}
* & * & 0 \\
* & 0 & * \\
* & 0 & 0
\end{array}\right] \quad \mathcal{A}_{3,3}=\left[\begin{array}{lll}
0 & * & 0 \\
* & * & * \\
* & 0 & 0
\end{array}\right] \quad \mathcal{A}_{3,4}=\left[\begin{array}{lll}
0 & * & 0 \\
* & 0 & * \\
* & 0 & *
\end{array}\right] \\
& \mathcal{A}_{3,5}=\left[\begin{array}{lll}
* & * & 0 \\
* & * & * \\
* & 0 & 0
\end{array}\right] \quad \mathcal{A}_{3,6}=\left[\begin{array}{lll}
* & * & 0 \\
* & 0 & * \\
* & 0 & *
\end{array}\right] \quad \mathcal{A}_{3,7}=\left[\begin{array}{lll}
0 & * & 0 \\
* & * & * \\
* & 0 & *
\end{array}\right] \quad \mathcal{A}_{3,8}=\left[\begin{array}{lll}
* & * & 0 \\
* & * & * \\
* & 0 & *
\end{array}\right] .
\end{aligned}
$$

Matrices $\mathcal{A}_{3, j}$ for $j=1, \ldots, 5$ are not potentially nilpotent over any field by Corollary 3.7. The matrices $\mathcal{A}_{3,6}$ and $\mathcal{A}_{3,7}$ are potentially nilpotent over any field $\mathbb{F}$ with realizations

$$
\left[\begin{array}{ccc}
-1_{\mathbb{F}} & -1_{\mathbb{F}} & 0 \\
1_{\mathbb{F}} & 0 & 1_{\mathbb{F}} \\
1_{\mathbb{F}} & 0 & 1_{\mathbb{F}}
\end{array}\right] \text { and }\left[\begin{array}{ccc}
0 & -1_{\mathbb{F}} & 0 \\
1_{\mathbb{F}} & -1_{\mathbb{F}} & 1_{\mathbb{F}} \\
1_{\mathbb{F}} & 0 & 1_{\mathbb{F}}
\end{array}\right]
$$

respectively. Finally, the pattern $\mathcal{A}_{3,8}$ cannot be potentially nilpotent over $\mathbb{Z}_{2}$ by Lemma 5.2 (a). Also, this pattern is not nilpotent over $\mathbb{Z}_{3}$; again, we use a Gröbner basis of $I_{\mathcal{A}_{3,8}}$ :

$$
\begin{gathered}
\left\{z_{1,1}+z_{2,2}+z_{3,3}, \quad z_{1,2} z_{2,3}+z_{2,2}^{2}+z_{2,2} z_{3,3}+z_{3,3}^{2}, \quad z_{1,2} z_{2,3} z_{3,1}+z_{3,3}^{3},\right. \\
\left.z_{2,2}^{2} z_{2,3} z_{3,1}+z_{2,2} z_{2,3} z_{3,1} z_{3,3}+z_{2,3} z_{3,1} z_{3,3}^{2}-z_{2,1} z_{3,3}^{3}\right\} .
\end{gathered}
$$

By Lemma 5.2 (c), the first polynomial implies $z_{1,1}=z_{2,2}=z_{3,3}=1_{\mathbb{F}}$ or $2_{\mathbb{F}}$. In the first case, the second polynomial reduces to $z_{1,2} z_{2,3}+1_{\mathbb{F}}+1_{\mathbb{F}}+1_{\mathbb{F}}=z_{1,2} z_{2,3}$ which is nonzero in $\mathbb{Z}_{3}$. Similarly, in the second case, the second polynomial equation becomes $z_{1,2} z_{2,3}+4_{\mathbb{F}}+4_{\mathbb{F}}+4_{\mathbb{F}}=z_{1,2} z_{2,3} \neq 0$. If $p \neq 2,3$, this pattern is potentially nilpotent with realization

$$
\left[\begin{array}{ccc}
-2_{\mathbb{F}} & -1_{\mathbb{F}} & 0 \\
3_{\mathbb{F}} & 1_{\mathbb{F}} & 1_{\mathbb{F}} \\
1_{\mathbb{F}} & 0 & 1_{\mathbb{F}}
\end{array}\right]
$$

Case 4. The non-loop edges are $(1,2),(2,1),(2,3),(1,3)$, and $(3,1)$.

We need to consider eight znz-patterns:

$$
\mathcal{A}_{4,1}=\left[\begin{array}{lll}
0 & * & * \\
* & 0 & * \\
* & 0 & 0
\end{array}\right] \quad \mathcal{A}_{4,2}=\left[\begin{array}{ccc}
* & * & * \\
* & 0 & * \\
* & 0 & 0
\end{array}\right] \quad \mathcal{A}_{4,3}=\left[\begin{array}{lll}
0 & * & * \\
* & * & * \\
* & 0 & 0
\end{array}\right] \quad \mathcal{A}_{4,4}=\left[\begin{array}{lll}
0 & * & * \\
* & 0 & * \\
* & 0 & *
\end{array}\right]
$$




$$
\mathcal{A}_{4,5}=\left[\begin{array}{ccc}
* & * & * \\
* & * & * \\
* & 0 & 0
\end{array}\right] \quad \mathcal{A}_{4,6}=\left[\begin{array}{ccc}
* & * & * \\
* & 0 & * \\
* & 0 & *
\end{array}\right] \quad \mathcal{A}_{4,7}=\left[\begin{array}{lll}
0 & * & * \\
* & * & * \\
* & 0 & *
\end{array}\right] \quad \mathcal{A}_{4,8}=\left[\begin{array}{ccc}
* & * & * \\
* & * & * \\
* & 0 & *
\end{array}\right]
$$

We can use Corollary 3.7 to eliminate the znz-patterns $\mathcal{A}_{4, j}$ for $j=1, \ldots, 4$. The patterns $\mathcal{A}_{4, j}$ for $j=5, \ldots, 8$ fail to be potentially nilpotent over $\mathbb{F}=\mathbb{Z}_{2}$ by Lemma 5.2 . Indeed, the first three are eliminated by part $(b)$, while the last is eliminated by $(a)$.

The matrices $\mathcal{A}_{4,5}$ and $\mathcal{A}_{4,6}$ are potentially nilpotent over any $\mathbb{F}=\mathbb{Z}_{p}$ with $p \neq 2$ with realizations

$$
\left[\begin{array}{ccc}
1_{\mathbb{F}} & 1_{\mathbb{F}} & 1_{\mathbb{F}} \\
1_{\mathbb{F}} & -1_{\mathbb{F}} & -1_{\mathbb{F}} \\
2_{\mathbb{F}} & 0 & 0
\end{array}\right] \text { and }\left[\begin{array}{ccc}
1_{\mathbb{F}} & 1_{\mathbb{F}} & 1_{\mathbb{F}} \\
1_{\mathbb{F}} & 0 & 2_{\mathbb{F}}^{-1} \\
-2_{\mathbb{F}} & 0 & -1_{\mathbb{F}}
\end{array}\right] \text { respectively. }
$$

When $p=3$, two of the polynomials in the Gröbner basis of $I_{\mathcal{A}_{4,7}}$ are $z_{1,2} z_{2,1}+$ $z_{3,1} z_{1,3}+z_{3,3}^{2}$ and $z_{1,2} z_{2,3} z_{3,1}-z_{1,3} z_{3,1} z_{3,3}+z_{3,3}^{3}$. By Lemma $5.2(\mathrm{c})$, the first polynomial can only equal zero if $z_{1,2} z_{2,1}=z_{3,1} z_{1,3}=z_{3,3}^{2}$ in $\mathbb{Z}_{3}$. Since $z_{3,3} \neq 0$, we will always have $z_{3,3}^{2}=1_{\mathbb{F}}$ in $\mathbb{Z}_{3}$. Hence $z_{1,3} z_{3,1}=1$ and the second polynomial reduces to $z_{1,2} z_{2,3} z_{3,1} \neq 0$ So, $\mathcal{A}_{4,7}$ is not potentially nilpotent over $\mathbb{Z}_{3}$. However, when $p \neq 3$, we have the realization

$$
\left[\begin{array}{ccc}
0 & -2_{\mathbb{F}} & 1_{\mathbb{F}} \\
1_{\mathbb{F}} & -1_{\mathbb{F}} & 3_{\mathbb{F}} \cdot 2_{\mathbb{F}}^{-1} \\
1_{\mathbb{F}} & 0 & 1_{\mathbb{F}}
\end{array}\right]
$$

The last pattern $\mathcal{A}_{4,8}$ is potentially nilpotent over $\mathbb{Z}_{p}$ for any prime $p \geq 3$ with realization:

$$
\left[\begin{array}{ccc}
-2_{\mathbb{F}} & -4_{\mathbb{F}} & 1_{\mathbb{F}} \\
1_{\mathbb{F}} & 1_{\mathbb{F}} & 4_{\mathbb{F}}^{-1} \\
1_{\mathbb{F}} & 0 & 1_{\mathbb{F}}
\end{array}\right]
$$

Case 5. The non-loop edges are $(1,2),(2,1),(3,2),(2,3),(1,3)$, and $(3,1)$.

We now need to consider the remaining four irreducible znz-patterns:

$$
\mathcal{A}_{5,1}=\left[\begin{array}{ccc}
0 & * & * \\
* & 0 & * \\
* & * & 0
\end{array}\right] \quad \mathcal{A}_{5,2}=\left[\begin{array}{lll}
* & * & * \\
* & 0 & * \\
* & * & 0
\end{array}\right] \quad \mathcal{A}_{5,3}=\left[\begin{array}{ccc}
* & * & * \\
* & * & * \\
* & * & 0
\end{array}\right] \quad \mathcal{A}_{5,4}=\left[\begin{array}{ccc}
* & * & * \\
* & * & * \\
* & * & *
\end{array}\right] \text {. }
$$

Pattern $\mathcal{A}_{5,2}$ is not potentially nilpotent over any $\mathbb{F}$ by Corollary 3.7. Also, by Lemma 5.2 (a), the pattern $\mathcal{A}_{5,4}$ is not potentially nilpotent over $\mathbb{F}=\mathbb{Z}_{2}$.

For the pattern $\mathcal{A}_{5,1}$, we have $z_{1,2} z_{2,1}+z_{1,3} z_{1,3}+z_{2,3} z_{3,2} \in I_{\mathcal{A}_{5,1}}$. This has no 
nonzero solution in $\mathbb{Z}_{2}$. When $p \neq 2$, one can use the realization:

$$
\left[\begin{array}{ccc}
0 & -1_{\mathbb{F}} & 1_{\mathbb{F}} \\
4_{\mathbb{F}} & 0 & 2_{\mathbb{F}} \\
2_{\mathbb{F}} & 1_{\mathbb{F}} & 0
\end{array}\right] .
$$

The pattern $\mathcal{A}_{5,3}$ is potentially nilpotent over any $\mathbb{F}=\mathbb{Z}_{p}$ with realization:

$$
\left[\begin{array}{ccc}
1_{\mathbb{F}} & 1_{\mathbb{F}} & 1_{\mathbb{F}} \\
-1_{\mathbb{F}} & -1_{\mathbb{F}} & -1_{\mathbb{F}} \\
1_{\mathbb{F}} & 1_{\mathbb{F}} & 0
\end{array}\right] .
$$

Finally, for any $p \geq 3$, the pattern $\mathcal{A}_{5,4}$ is potentially nilpotent over $\mathbb{F}=\mathbb{Z}_{p}$; indeed, one such realization is

$$
\left[\begin{array}{ccc}
1_{\mathbb{F}} & 1_{\mathbb{F}} & 1_{\mathbb{F}} \\
1_{\mathbb{F}} & 1_{\mathbb{F}} & 1_{\mathbb{F}} \\
-2_{\mathbb{F}} & -2_{\mathbb{F}} & -2_{\mathbb{F}}
\end{array}\right] .
$$

REMARK 5.4. We point out three interesting facts that arise from this classification. First, all the irreducible patterns that are not potentially nilpotent over any $\mathbb{Z}_{p}$ are in fact not potentially nilpotent over any field $\mathbb{F}$. As a consequence, to determine which irreducible patterns are potentially nilpotent over $\mathbb{F}=\mathbb{R}$, it suffices to consider only the irreducible patterns that appear in the statement of Theorem 5.3. Moreover, the realizations given in the proof of Theorem 5.3 show that all of these irreducible patterns are potentially nilpotent over $\mathbb{R}$ except the pattern in Case 4 . Second, if $\mathcal{A}$ is potentially nilpotent over $\mathbb{Z}_{p}$, it does not necessarily follow that any superpattern of $\mathcal{A}$ continues to be potentially nilpotent over $\mathbb{Z}_{p}$. And third, notice that none of the cases in Case 4 are potentially nilpotent over $\mathbb{Z}_{2}$. This lends itself to a natural question: what digraphs $D(\mathcal{A})$ have the property that $\mathcal{A}$ fails to be potentially nilpotent over some field $\mathbb{F}$, regardless of the placement of the loops?

Acknowledgments. Many of these results were inspired through computer experiments using CoCoA [4] and Macaulay 2 [12]. The second author thanks Redeemer University College for its hospitality while working on this project, while both authors thank Natalie Campbell for helpful discussions.

\section{REFERENCES}

[1] E. Bodine. Spectrally Arbitrary Zero-Nonzero Patterns over Finite Fields. Western Canada Linear Algebra Meeting, University of Regina, May 30, 2008.

[2] T. Britz, J.J. McDonald, D.D. Olesky, and P. van den Driessche. Minimal spectrally arbitrary patterns. SIAM Journal of Matrix Analysis and Applications, 26:257-271, 2004. 
[3] R. A. Brualdi. From the Editor-in-Chief. Linear Algebra and its Applications, 420:728, 2007.

[4] CoCoATeam. CoCoA: a system for doing Computations in Commutative Algebra. http://cocoa.dima.unige.it

[5] L. Corpuz and J.J. McDonald. Spectrally arbitrary zero-nonzero patterns of order 4. Linear and Multilinear Algebra, 55:249-273, 2007.

[6] D. Cox, J. Little, and D. O'Shea. Ideals, Varieties, and Algorithms. An Introduction to Computational Algebraic Geometry and Commutative Algebra. Undergraduate Texts in Mathematics. Springer, New York, 1992.

[7] L.M. DeAlba, I.R. Hentzel, L. Hogben, J. McDonald, R. Mikkelson, O. Pryporova, B. Shader, and K.N. Vander Meulen. Spectrally arbitrary patterns: reducibility and the $2 n$ conjecture for $n=5$. Linear Algebra and its Applications, 423:262-276, 2007.

[8] J.H. Drew, C.R. Johnson, D.D Olesky, and P. van den Driessche. Spectrally arbitrary patterns. Linear Algebra and its Applications, 308:121-137, 2000.

[9] C. Eschenbach and C.R. Johnson. Several open problems in qualitative matrix theory involving eigenvalue distribution. Linear and Multilinear Algebra, 24:79-80, 1988.

[10] C. Eschenbach and Z. Li. Potentially nilpotent sign pattern matrices. Linear Algebra and its Applications, 299:81-99, 1999.

[11] Y. Gao, Z. Li and Y. Shao. Sign patterns allowing nilpotence of index 3. Linear Algebra and its Applications, 424:55-70, 2007.

[12] D. R. Grayson and M. E. Stillman. Macaulay 2, a software system for research in algebraic geometry. http://www.math.uiuc.edu/Macaulay2/

[13] K. Kaphle. Spectrally arbitrary tree sign pattern matrices. M.Sc. Thesis, Georgia State University, 2006

[14] I.-J. Kim, D.D. Olesky, and P. van den Driessche. Inertially arbitrary sign patterns with no nilpotent realization. Linear Algebra and its Applications, 421:264-283, 2007.

[15] I.-J. Kim, D.D. Olesky, P. van den Driessche, H. van der Holst, and K.N. Vander Meulen. Generating potentially nilpotent full sign patterns. Electronic Journal of Linear Algebra, 18:162-175, 2009.

[16] R. Lidl and H. Niederreiter. Introduction to finite fields and their applications. Revision of the 1986 first edition. Cambridge University Press, Cambridge, 1994.

[17] G. MacGillivray, R.M. Tifenbach, and P. van den Driessche. Spectrally arbitrary star sign patterns. Linear Algebra and its Applications, 400:99-119, 2005.

[18] R. Pereira. Nilpotent matrices and spectrally arbitrary sign patterns. Electronic Journal of Linear Algebra, 16:232-236, 2007.

[19] B.L. Shader. Notes on the $2 n$-conjecture. http://www . aimath.org/pastworkshops/matrixspectrum.html

[20] L. Yeh. Sign pattern matrices that allow a nilpotent matrix. Bulletin of the Australian Mathematical Society, 53:189-196, 1996.

[21] A. Yielding. Spectrally Arbitrary Zero-Nonzero Patterns. Western Canada Linear Algebra Meeting, University of Regina, May 30, 2008. 